\title{
THE IMPORTANCE OF RESPONSIVE LOGO DESIGN ACROSS A WIDE RANGE OF DEVICES ON THE WEB
}

\author{
Aleksandar Mihajlović, \\ Jelena Gajić, \\ Jelena Stanković, \\ Milan Tair \\ Singidunum University, \\ 32 Danijelova Street, Belgrade, Serbia
}

\begin{abstract}
:
The aim of this paper is to clarify the significance of using responsive logos on web pages. It aims to bring a more flexible and contextual system of brand identification. This can be done by moving away from strict and inflexible design guidelines. The authors shall attempt to explain why logos in all its variations can be unambiguous memory hooks. Also, we emphasise that they can behave like many other elements in responsive web pages. Contemporary businesses demand adaptation of their content to many sophisticated communication platforms. Among these are the devices with different varying screen dimensions. A responsive logo can be used to build better user experiences across a wide range of devices, thus elevating brand freshness. Having in mind that logos are the key elements of corporate identity and brand positioning, it is mandatory to optimise it for every scenario. It is an element on par with colours or slogans. It can be simplified by applying responsive design principles to individual elements that make out the logo. By removing unnecessary parts in relation to the varying screen size, a clearer and much more readable logo can be arranged. We have built a model for applying responsive design to logos and performed experimental testing in the real environment.
\end{abstract}

Key words:

logo design, branding, responsive design, scalable vector graphics (SVG).

\section{INTRODUCTION}

Over the 25 year long history of the web, all images, including logos, have remained the most important pieces of information on the Internet. Unfortunately, they have not always been adaptable to varying screen sizes. Everything about them has been fixed, their size, format, etc. (Eric Portis, 2014). Over the years, various computer display resolution (specified as the width and height in pixels) have been used. With arrival of devices of different sizes (desktops, laptops, tablets, phablets and mobile phones), the need for change and adaptation has become constant. In 2001, the first responsive web design (RWD) site was introduced. RWD aims to provide methods for adapting the web page content for viewing and seamless user experience transition with minimum resizing, panning and scrolling, across a wide range of devices.

Also, RWD helps to create active content that adapts to the properties of the device used to render it to the user. Web pages are typically created using Hyper-Text Mark-Up Language (HTML) to mark-up their content which defines the structure of the very page. On the other hand, plain 
content is not sufficient on the modern web. Instead, Cascading Style-Sheet (CSS) code is used to define custom styling of web page structures, such as images, content blocks, sections, links, buttons, etc. The CSS language has evolved over time to provide many new features for styling web content and to provide ever more flexible, dynamic and definitions for fully customize design of page elements. One of the most powerful CSS properties are media queries. Media queries allow programmers to provide custom style-sheet definitions for elements of the web page when a certain set of criteria are met. These media queries can consist of a single criterion or, more frequently, a set of criteria such as that the device is a screen (a device where the user can see the rendered graphical representation of the web page) and that the minimum device width needs to be of a certain length (i.e. in pixels) for the CSS styles defined in that media query definitions to be used.

From the beginning, images have been the number one obstacle in implementing truly adaptable and operational responsive pages. Their inability to effectively adapt to both the constraints and affordances of the browsing context at hand was the key issue. In most scenarios, logos are simply scaled down to fit the available space in the resized context. This causes the logo to become unreadable and blurred.

\section{LOGO - VISUAL SIDE OF BRAND}

In the business world, logos are communication "touch points" linking companies to the stakeholders. The logo, as a promotional tool, (Ashworth \& Kavaratzis, 2009 ) is a part of intentional formal communication. Hildreth (2010) considers logos as identifiers, while Stock (2009) defines logos as straightforward applications of branding tools. The main identity component of functional brand (Kotler, 2003) - the logo, should be easily recognised and applied to various promotional platforms. This applies both to the physical and digital environment. It makes the brand visible (Kelly, 2016). Consumers and stakeholders come across visual stimuli (logo, colours, shapes, styles, taglines, typefaces, etc.) when interacting with brands. It indicates that the visual elements should be created correctly in order to make popular and needed brand association with customers (Philips et al., 2014).

Milton Glaser states that logos are gateways to brands (Kelly, 2016, p.8-9). Also, their main functions are to trigger perceptions, to create first-hand associations, to play a major role in the place-branding process and to be a quick form of communication. Snyder pointed out (1993) that logos represent visual signatures for brands.
These logos are responsible for building and maintaining brand's personality (Zakia \& Nadin, 1987). Logos are not only the means of capturing customers' awareness but are among the most important means of communication. This is due to the fact that customers are primarily exposed to them when establishing initial contact with companies (Cian et al., 2014).

Using flexible identity of logo enables strengthening and enhancing brand position. This helps uniting communication strategies visually. In a highly developed digital surrounding of contemporary businesses, it is mandatory to consider using it for the purposes of creating responsive logo design. The flexible identity of logo supports maximising logo visibility. This is achieved through various design applications moving beyond the rules that govern the common use of the logo. The flexible identity means that each touch point, such as any piece of the signage, the artefact, wall colour, etc. offers visual symbolism and the connection to the brand. This does not necessarily include the use of the logo itself (Kelly, 2016).

The importance of visual aspects of logo is recognised in communicating brand strategy and marketing practice. It requires investments in its (re)designing. For instance, the costs of logo design for the 2012 Olympics in London reached \$625.000. In 2008, Pepsi spent \$1 million. Furthermore, British Petroleum invested \$211 million for their logo redesign in the same year (Cian et al., 2014).

A brand has to be amicable and comprehensive by everyone at all touch points. Also, the introduction of digital portable devices calls for complete and continuous processes of adaptation of a range of visual brand elements for different marketing purposes. Digital platforms such as smart phones and social media encourage logo adaptation. This is required in order to achieve better flexibility and remain visible. Thus, responsive logo design must be applied carefully in favour of enhancing brand image and identity (Kelly, 2016).

\section{PROBLEM DEFINITION}

The aim of this paper is to clarify the importance of visual punctuation, present the responsive logo design concept and demonstrate the potential solution to one of the problems that companies on the web face today. Due to the increasing use of different types of devices, companies need to adjust their web sites for different resolutions and orientation. This is done by creating responsive web sites. Responsive design is made possible by means of media queries. As mentioned before, each media query consists of criteria that need to be met in order for the set of defined CSS styles to be applied. The 
first part of the query that makes a single criterion is the media type or the media device (Carey, 2013). After the media type is defined, a set of expressions follow. They can better define the circumstances when the media query definitions should take effect. These expressions can be used to recognize device properties and states, such as the width, orientation, colour depth, resolution, scanning output type for television screens and similar (Lewis \& Moscovitz, 2009).

Google also announced that responsive design would become a notable ranking factor in the Search engine results page (SERPs). Because of this, all companies on the market that wish to gain and/or retain competitive advantage take responsive design as an extremely important development objective. As Ofcom's Eighth International Communications Market Report (2014) shows, smart phones and tablet devices are equally important as desktop and laptop. This means that having a consistent strategy across all user platforms can be extremely effective. This trend is so prevalent that Google has begun boosting ratings of sites that are mobile friendly. This ranking applies for searches made from mobile devices.

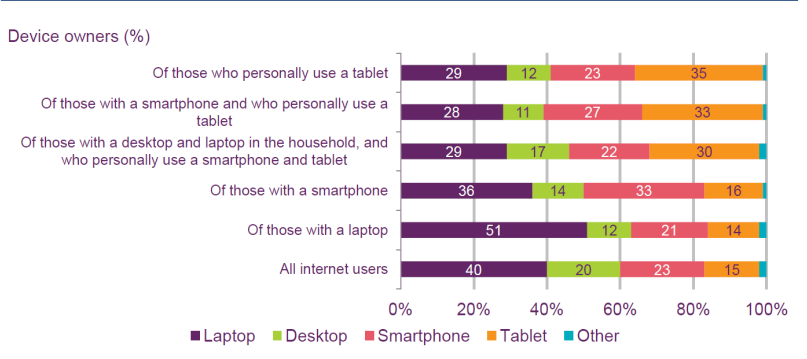

Graph 1. Most important devices for connecting to the Internet (Ofcom research, Q1, 2014)

In the research published by the Social media today, $83 \%$ of mobile users say that a seamless experience across all devices is very important (Miles, 2015). The assumption that a large number of companies have already responded to the market demands is wrong. The real situation is totally different. When it comes to the top 100 sites analysed, only $11.8 \%$ of these sites use responsive web design (Meunier, 2015). There is an issue of large number of these responsive websites. The problem is that the brand symbol or logo, simply often does not, or cannot, respond well to changes in a way that it is displayed. The importance of this problem is large, considering that this is the key element in corporate visual communication. Therefore, it must not be neglected. Nevertheless, many companies and their web designers do not consider this issue. In some cases, they were prohibited from dealing with it in case of more conservative corporate cultures.
The market demands for responsive web design are creating an ever growing pressure to adapt logos to smaller screen sizes. In most cases, the logo is just shrunk to fit the available space. This approach can work for rectangular logos with simple and minimalistic appearances. On the other hand, if the composition or proportion of a logo is different, simply reducing its size can make smaller details unrecognisable and small type unreadable. This can be overcome by adopting the concept of responsive logo design for RWD.

\section{ADVANTAGES AND DISADVANTAGES OF RESPONSIVE WEB DESIGN}

With the emergence of hand-held computers, such as PDAs and early tablet computers, developers have struggled to adapt the concurrent user interface philosophy to the new platform (Robertson, Wharton, \& Ashwo, 1996). Unlike these scenarios, where one user interface is designed for the purpose of a specific device or screen type, responsive design aims to provide the same content for multiple devices without having to take the user to a different web page where different design definitions are used (Kuo-Ying, 2009). Responsive design concepts are employed mostly on the web. However, modern mobile applications employ their own design pattern for responsive user interfaces (Lehtimaki, 2012). These design patterns differ from the concept of responsive web page design, in the way that they are adapted for rendering on mobile devices. Nevertheless, the web page design that targets even mobile phones continues to gain popularity (Google Trends, 2016). Disadvantages of responsive design patterns are related to the fact that they are used to adapt the content to smaller screen sizes. When doing this, certain information may be lost and some features, such as navigation are made more complicated. Normally, on desktop sized devices, web pages feature a single click menu, where submenus can be uncovered upon hovered above their parent menu item. Finally, only one menu item is activated by clicking on it. With mobile phones, hovering is not yet practical. In such cases, web sites adapt the menu to be expandable upon click in order to uncover children items. This, in turn, requires more interaction from the user in order to properly navigate the web page. Another disadvantageous consequence of smaller screen size is the need to scroll through the content. Originally, web pages that have their content optimised for desktop screens have more content which, when condensed to mobile screens, tends to expand the virtual scrollable screen to greater lengths requiring even more user engagement in order to properly review the content. This can sometimes 
have profoundly negative effects on the experience of mobile phone users viewing the responsive web page, as explained by (Lestari, Hardianto, \& Ni, 2014) and (Hussain \& Mkpojiogu, 2015). Regardless of this fact and based on the positive trends mentioned before, the advantages of responsive design outweigh its disadvantages. New web standards allow for new extensions of the responsive design. Among these is the concept of creating responsive modules. This, so called modular responsive design, allows for certain parts of the user interface to be adaptive to their own immediate surroundings. This happens to be unrelated to the context of the page and its surrounding device screen (Wiener, Ekholm, \& Haller, 2015).

\section{PROPOSED SOLUTION FOR RESPONSIVE LOGO}

As we have discussed in the previous sections, logos are usually simply shrunk to fit the available space. Because of this, they are not responsive. The idea behind responsive web design is that its layout should be readjusted according to the screen on which it is being viewed. At the same time, it should be readable on a 4-inch smart phone, just as on a 27 -inch monitor. Logos should act in the same way. They should change shape, simplify and lose words or other fragments. In other words, they should respond to the surrounding context.

To solve this problem it takes a different approach in understanding the boundaries of logos. Companies can revise their logos to become simple and scalable. Elsewise, they can change the way they implement their logos. Instead of using up valuable screen space with an unresponsive logo that becomes indistinct and unrecognisable, a better solution is to provide responsive logo that can adapt to varying sizes.

A few years back, a London-based designer, Joe Harrison, famously experimented with responsive logos. $\mathrm{He}$ launched a concept which took the iconic logos of big brands and put them through their responsive paces (Harrison, 2015). For example, the "Walt Disney Pictures" logo (Cass, 2015) changes are shown in Image 1, as the browser windows resizes. The logo first loses its castle and becomes a type, then it shortens to Disney, before finally shrinking into a whimsical cursive letter " $D$ ".

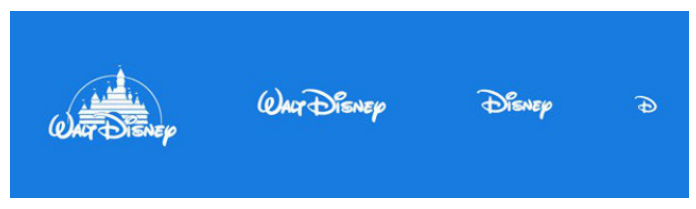

Image 1. Example of responsive logo - „Walt Disney Pictures" (Cass, 2015)

\section{Branding guidelines}

Well-designed identities often allow logos to be displayed in different variations depending on the context. Usually, they are presented and outlined in a branding guidelines document. These guidelines provide a rule on proper implementation of logo within certain size constraints. In cases of responsive logo, guidelines should specifically provide alternate logo formats that could be used in smaller areas.

Another example is shown in for Singidunum University's logo in Image 2. A well-defined branding guideline provides an alternate format of logo that could be used in smaller areas.

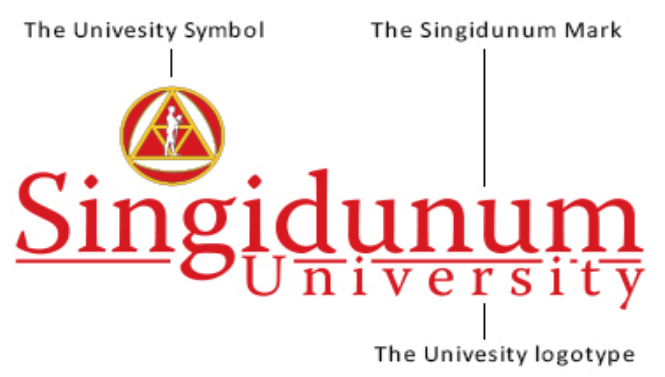

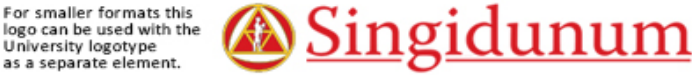

Image 2. An example of an alternate mode of Singidunum University's logo

The small version of the logo, displayed in Image 2, has the logotype removed and the mark expanded to match the height of the symbol. See Image 3 that compares the two modes of the logo in a condensed header on a $320 \mathrm{px}$ wide small screen. It is evident that the bottom logo is more readable and less untidy.

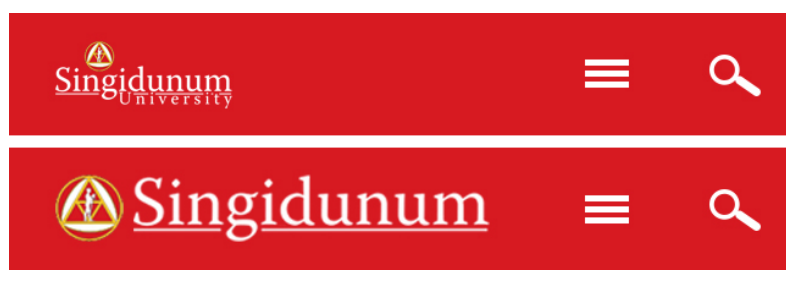

Image 3. Examples of logos displayed on a 320px wide small screen

Brand recognition and visual identification will not be lost, if adjustments are made because of physical limitations. Flexibility can support and enhance the identity as a whole. 


\section{Reduction of details}

In rare cases, a detailed logo appears. By reducing the level of details at small sizes, logo is simplified and readability can be greatly enhanced. For that purpose, detailed shapes can be simplified and trimmed. Thin lines can be made thicker; outline elements could be inverted and filled in, etc. For each variety of logo, the level of detail is progressively reduced. This can be done by reducing its size, which greatly enhances the readability of logo at small sizes.

\section{Implementation of RWD and usage of image sprites}

Technical implementation of responsive design relies on modern Internet and web technologies. Among these, the most important is the web browsers' support for the advanced CSS version 3 language features. Besides original CSS features that allowed for limited responsive implementations even before CSS version 3, the new standard has introduced a number of new devices or media types.

A code snippet, which includes a CSS code with the media query feature shown below, illustrates a method of creating a responsive logo for four different screen resolution widths.

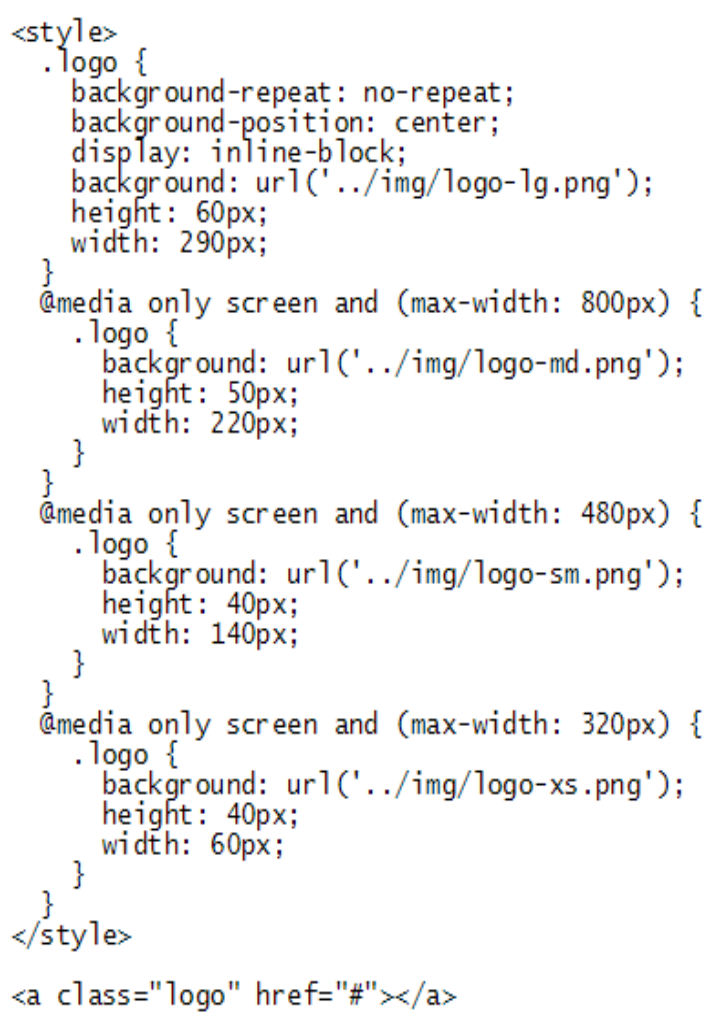

Listing 1. A code snippet that includes a media query CSS rule set that adds support for a responsive logo for four different screen resolution widths
The techniques commonly used for displaying different images at different breakpoints are image sprites. A sprite is a collection of images stored within a single file. Web sites with many images require more time to load. At the same time, they generate multiple server requests. By using image sprites, loading time and the server request count is reduced. This results in saving bandwidth (Bowers, Synodinos, \& Sumner, 2011). However, it yields the need to create sprite images with each variation of the logo, and potentially additional images for each targeted pixel density.

Another option is to use an adaptive, multi-layered image format with layers set to appear when overlaid over different backgrounds and sizes. We are currently experimenting with this concept. It will support the needs of new responsive image techniques. However, it is still in experimental phase. Currently, it only includes implementations for adapting the logo to varying background colours and brightness intensities.

\section{Use of Scalable Vector Graphics (SVG) images}

With the growing need for resolution independent graphics that consumes the Internet, the necessity to use SVG has appeared. SVG is a file format that scales without losing definition. It gives us resolution independence and true single file implementations.

\section{CONCLUSION}

As described in this paper, the existing solutions that aim to solve the issue of logo adaptability are usually sufficient. However, we have identified that there are practical challenges for implementing a truly responsive logo within a responsive web page. In order to support multiple screen resolutions and screen densities, logos must be remade to comprise of modular units that, on their own, sufficiently represent and help identify with the brand they belong to. These units should be simple in terms of graphical design in order to be readable and recognisable even when displayed in very small sizes. We reproduce a responsive logo by means of CSS media queries implementation and prove the concept. Also, we propose the use of componential logos made in the vector format, preferably SVG. Finally, our conclusion is that there is still space for future work. This is mainly because the current responsive logo concepts depend on the web page dimensions. We suggest expanding the concept to include other properties of the surrounding context, such as the background of the logo. Currently, 
designers and web developers need to take into account the underplaying background of the logo and need to display a light or dark logo variant based on that.

\section{FUTURE WORK}

In the future, the authors will develop a model that implements a responsive logo for vector and raster images in a multi-layer image file format that supports additional meta-data. That will ensure the proper use of the responsive logo according to the predefined rules, and it will be done automatically. That will result in a decreasing number of errors when applying the logo by third parties. Also, the authors shall devote particular attention in their future work to conducting experimental research that will expose business clients and consumers to responsive logos on the web and examine their experience and reactions to modified design logo solutions.

\section{REFERENCES}

Ashworth, G., Kavaratzis, M. (2009) Beyond the logo: Brandmanagement for cities. Journal of Brand Management 16(8): 520-531.

Bowers, M., Synodinos, D., Sumner, V. (2011) Pro HTML5 and CSS3 Design Patterns, 308-311.

Carey, P. (2013). New Perspectives on HTML, CSS, and $X M L$, Comprehensive. Cengage Learning.

Cass, J. (2015, November 19). Retrieved from Logos in a Responsive Design World: justcreative. com/2015/11/19/the-problem-of-the-logo-in-aresponsive-design-world

Cian, L., Krishna, A., Elder, R.S. (2014). The Logo Moves Me. Journal of Marketing Research. American Marketing Society, Vol. LI, April, p. 184-197

Google Trends. (2016, March 29). Retrieved March 29, 2016, from Google Trends: google.com/trends/exp lore?q="responsive+web+design"

Harrison, J. (2015). Responsive Logos. Retrieved from responsivelogos.co.uk

Hildreth, J. (2010). Place branding: A view at arm's length. PlaceBranding and Public Diplomacy 6(1): 27-35

Hussain, A., \& Mkpojiogu, E. O. (2015). The effect of responsive web design on the user experience with laptop and smartphone devices. Jurnal Teknologi, 41-47.

Kelly, M. (2016). Analysing the complex relationship between logo and brand, Place Branding and Public Diplomacy 1-16., Macmillan Publishers Ltd. retrived in March, 2016 from palgrave-journals.com/pb

Kotler, P. (2003). Marketing Management. New Jersey: Pearson Education
Kuo-Ying, H. (2009). Challenges in Human-Computer Interaction Design for Mobile Devices. Proceedings of the World Congress on Engineering and Computer Science. San Francisco: WCECS.

Lehtimaki, J. (2012). Smashing Android UI: Responsive User Interfaces and Design Patterns for Android Phones and Tablets. John Wiley \& Sons.

Lestari, D. M., Hardianto, D., \& Ni, A. (2014). Analysis of User Experience Quality on Responsive Web Design from its Informative Perspective. International Journal of Software Engineering and Its Applications, (pp. 53-62).

Lewis, J., \& Moscovitz, M. (2009). AdvancED CSS. Apress. Meunier, B. (2015, January 15). 82\% Of Sites Use Responsive Web Design In 2015? Try 11.8\%. Retrieved from marketingland.com/82-sites-use-responsive-webdesign-2015-try-11-8-114050

Miles, G. (2015, November 13). Retrieved from 8 Mobile Marketing Stats To Help You Plan For 2016: socialmediatoday.com/marketing/8-mobile-marketingstats-help-you-plan-2016

Ofcom research, Q1. (2014). Retrieved from Ofcom's Eighth International Communications Market Report: stakeholders.ofcom.org.uk/binaries/research/ cmr/cmr14/2014_UK_CMR.pdf

Philips, J.B., McQuarrie, F.E., Griffin, G.W.(2014). How Visual Brand Identity Shapes Consumer Response, Psychology and Marketing, Vol. 31(3): 225-236.

Portis, E. (2014). Responsive Images Done Right: A Guide To And srcset. Retrieved 2016, from smashingmagazine.com/2014/05/responsive-images-done-rightguide-picture-srcset

Robertson, S., Wharton, C., \& Ashwo, C. (1996). Dual Device User Interface Design: PDAs and Interactive Television. CHI' 96 Proceedings of the SIGCHI Conference on Human Factors in Computing Systems, (pp. 79-86). New York.

Snyder, A. (1993). Branding: Coming up for more air, Brandweek, 34, December 6, p. 24-28.

Stock, F. (2009) Identity, image and brand: A conceptualframework. Place Branding and Public Diplomacy 5(2): 118-125.

Web Technology Surveys. (2016). Usage of image file formats for websites. Retrieved 01 11, 2016, from w3techs.com/technologies/overview/image_for$\mathrm{mat} / \mathrm{al}$

Wiener, L., Ekholm, T., \& Haller, P. (2015). Modular Responsive Web Design using Element Queries. Computing Research Repository, abs/1511.01223.

Zakia, R.D., Nadin, M. (1987) Semiotics, advertising and marketing. Journal of Consumer Marketing, $4(2)$, p. $5-12$. 